\title{
Effect and Neuroimaging Mechanism of Electroacupuncture for Vascular Cognitive Impairment No Dementia: Study Protocol for a Randomized, Assessor-Blind, Controlled Clinical Trial
}

\author{
Ruizhu Lin, ${ }^{1,2}$ Jia Huang, ${ }^{1}$ Jianfeng Xu, ${ }^{2}$ Jing Tao, ${ }^{1}$ Ying Xu, ${ }^{1}$ Jiao Liu, ${ }^{1}$ Weilin Liu, \\ Shengxiang Liang, ${ }^{1}$ Minguang Yang, ${ }^{1}$ and Lidian Chen $\mathbb{D}^{3}$ \\ ${ }^{1}$ Rehabilitation Medicine College, Fujian University of Traditional Chinese Medicine, Fuzhou 350122, China \\ ${ }^{2}$ General Hospital of Ningxia Medical University, Yinchuan, Ningxia, China \\ ${ }^{3}$ Fujian University of Traditional Chinese Medicine, Fuzhou 350122, China \\ Correspondence should be addressed to Lidian Chen; 13519217785@163.com
}

Received 12 September 2019; Revised 1 February 2020; Accepted 5 February 2020; Published 26 February 2020

Academic Editor: Gerhard Litscher

Copyright (๑ 2020 Ruizhu Lin et al. This is an open access article distributed under the Creative Commons Attribution License, which permits unrestricted use, distribution, and reproduction in any medium, provided the original work is properly cited.

\begin{abstract}
Vascular cognitive impairment no dementia (VCIND) is likely to develop into vascular dementia (VD) without intervention. The clinical efficacy of electroacupuncture (EA) for VCIND has been previously demonstrated. However, the neuroimaging mechanism of EA for VCIND has not been elucidated clearly. This trial is designed to provide solid evidence for the efficacy and neuroimaging mechanism of EA treatment for patients with VCIND. This ongoing study is an assessor-blind, parallel-group, randomized controlled trial. 140 eligible subjects will be recruited from the General Hospital of Ningxia Medical University and randomized into either the electroacupuncture (EA) group or the control group (CG). All subjects will receive basic treatment, and participants in the CG will receive health education performed weekly. Except for basic treatment and health education, participants in the EA group will receive treatment 5 times per week for a total of 40 sessions over 8 weeks. The primary outcome in this study is Montreal Cognitive Assessment (MoCA), and the secondary outcomes are Auditory Verbal Learning Test (AVLT), Stroop color-naming condition (STROOP), Rey-Osterrieth Complex Graphics Testing, and resting-state functional magnetic resonance imaging (rs-fMRI). All of the outcome measures will be assessed at baseline and 8 weeks of intervention. The medical abstraction of adverse events will be done at each visit. The results of this trial will demonstrate the efficacy and neuroimaging mechanism of EA treatment for VCIND, thus supporting EA treatment as an ideal choice for VCIND treatment. The trial was registered at the Chinese Clinical Trial Registry on 28 July 2018 (ChiCTR1800017398).
\end{abstract}

\section{Introduction}

Vascular cognitive impairment (VCI) is cognitive dysfunction caused by cerebrovascular disease or factor, ranging from mild cognitive dysfunction (vascular cognitive impairment no dementia, VCIND) to overt dementia (vascular dementia, VD) [1]. Therefore, VCIND constitutes an intermediate stage between normal aging and vascular dementia [2]. VCIND manifests mainly cognitive deficits in at least one cognitive domain without impairment of activities of daily living (ADL) [3,4]. A widely shared view is that up to
$46 \%$ of patients with VCIND progress to VD after 5 years of diagnosis are without treatment [5]. Therefore, the early intervention of VCI, particularly VCIND, which would greatly reduce the chances of developing VD in later life, is particularly important.

In fact, previous research on EA has demonstrated that EA is an effective treatment to improve cognitive function by VCIND [6, 7]. One study revealed that, after 8 weeks of scalp EA treatment, the Mini-Mental State Examination score increased from $25.1 \pm 0.9$ to $28.4 \pm 1.4$, and the picture recognition increased from $10.1 \pm 3.1$ to $12.5 \pm 3.1$. Therefore, 
opposed to conventional treatment, EA is a promising and simple method of improving the cognitive function of the cognitive impairment patients [8]. In an animal model of cognition disorder, it has been supported that EA can improve memory and reduce neuroinflammation associated with dementia, which means to have neuroprotective effects for the brain [9]. However, the mechanism of EA in the treatment of VCIND remains unclear, especially in neuroimaging. Currently, rs-fMRI has been recognized as one of the most promising techniques for neuroscientists to decode brain activity, especially for elderly or patients with cognitive impairment $[10,11]$. Neuroimaging studies have verified that the human brain has a default functional network (DMN) at rest [12]. Previous studies have demonstrated that cognitive disorders in subcortical vascular cognitive impairment (SVCI) are associated with alteration in resting DMN [13].

On the other hand, many studies have indicated that acupuncture regulates the activity of some cortical and subcortical brain regions, including the alteration of DMN $[14,15]$. Therefore, we hypothesize that EA may improve the cognitive function of VCIND due to changes with DMN. Through this proposed randomized controlled trial (RCT), we plan to explore the potential mechanism of EA in improving cognitive function in patients with VCIND.

Therefore, during this trial, our main aim is to assess the effect of EA on cognition function for VCIND. Our second aim will be to explore the neuroimaging mechanism after EA treatment on VCIND.

\section{Methods}

2.1. Study Design and Setting. This study is an assessor-blind parallel-group RCT to explore the clinical efficacy and neuroimaging mechanism of EA for improving the cognitive function of VCIND patients. It will be performed in the General Hospital of Ningxia Medical University. A total of 140 eligible subjects will be randomly divided into 2 groups, namely, (1) EA group and (2) CG group, in a ratio of $1: 1$. All subjects will receive basic treatment with essential drugs, including hypotension, hypoglycemics, and antiplatelet and hypolipidemic drugs. In the CG group, they will only receive health education once a week, a total of 8 times. Except for basic treatment and health education, participants in the EA group will receive EA treatment that will be performed for 30 min per day and 5 days per week for 8 weeks. Operators with more than 5 years of clinical experience will perform the interventions. The 8-week intervention will be conducted. Outcome measurements include Montreal Cognitive Assessment (MoCA), Auditory Verbal Learning Test (AVLT), Test of Attentional Performance (TAP), Stroop color-naming condition (STROOP), Rey-Osterrieth Complex Graphics Testing (ROCFT), resting-state functional MRI (rs-fMRI), and adverse events. The planned flowchart for this trial is shown in Figure 1. The time point of assessment is provided in Table 1. Ethical approval has been provided with the Ethics Committee of the General Hospital of Ningxia Medical University in April 2018 (Additional file 1). Besides, General Hospital of Ningxia Medical University is also the project undertaking unit, which is responsible for coordinating

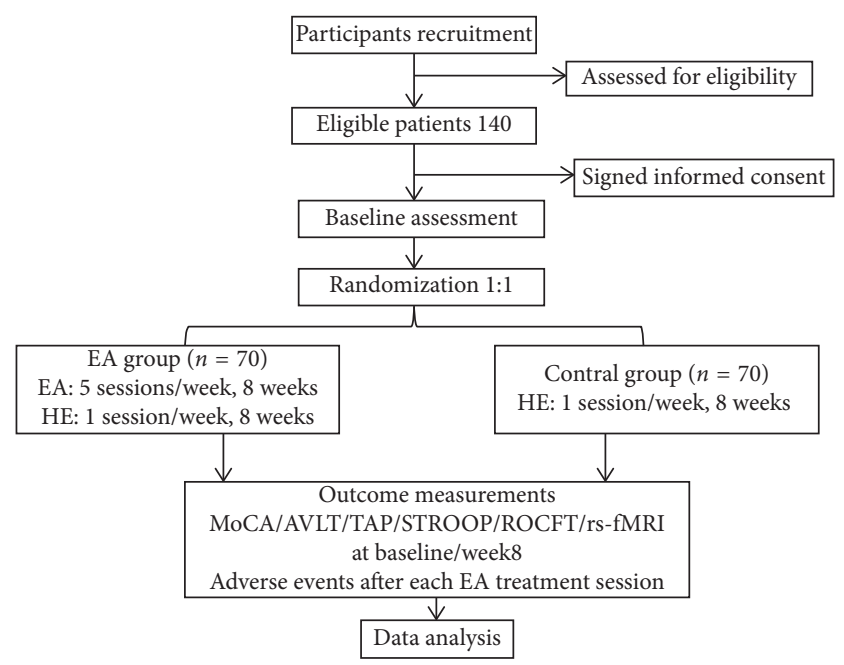

FIGURE 1: The planned flowchart of the trail. EA: electroacupuncture; HE: health education; MOCA: Montreal Cognitive Assessment; AVLT: Auditory Verbal Learning Test; TAP: Test of Attentional Performance; STROOP: Stroop color-naming condition; ROCFT: Rey-Osterrieth Complex Graphics Testing; rs-fMRI: resting-state functional MRI.

TABle 1: The study schedule for enrollment, interventions, and assessments.

\begin{tabular}{lccc}
\hline Items & $\begin{array}{c}\text { Before } \\
\text { enrollment } \\
-2 \text { to }-1 \text { week }\end{array}$ & $\begin{array}{c}\text { Intervention } \\
\text { period 1-8 } \\
\text { weeks }\end{array}$ & $\begin{array}{c}\text { Outcome } \\
\text { assessment 9 } \\
\text { weeks }\end{array}$ \\
\hline $\begin{array}{l}\text { Inclusion criteria } \\
\text { Exclusion criteria }\end{array}$ & $\times$ & & \\
Informed consent & $\times$ & & \\
Baseline & $\times$ & & \\
Randomization & $\times$ & & \\
and allocation & & & \\
Intervention & & & \\
Baker Depression & $\times$ & & $\times$ \\
Scale test & $\times$ & & $\times$ \\
MoCA & $\times$ & & $\times$ \\
AVLT & $\times$ & & \\
TAP & $\times$ & & \\
STROOP & $\times$ & & \\
ROCFT & $\times$ & & \\
rs-fMRI & & & \\
Adverse events & & & \\
Reasons of & & & \\
dropout and & & & \\
withdrawals & & &
\end{tabular}

the activities of all departments (e.g., trial registries, researcher training, informed consent of the participants, and data management). If there is an important protocol to be modified, it will notify the relevant parties to hold a coordination meeting. This protocol follows Standard Protocol Items: Recommendations for Interventional Trials (SPIRIT) (see Additional File 1 in Supplementary Materials) [16].

2.2. Sample Size Calculation. In this procedure, the total score of the Montreal Cognitive Assessment Scale was invoked as the main effect index. The effect value was 0.9 in the 
treatment group and 0.725 in the control group [17], 0.8 in power and 0.05 in alpha. The sample size was estimated at PASS 24.0 software to be 116 cases, i.e., 58 cases in each group. Allowing $20 \%$ dropout rate, the total sample size was expected to be 140 patients (70 in each group).

2.3. Participants and Recruitment. A total of 140 righthanded participants will be recruited from community VCIND patients in Yinchuan, Ningxia Hui Autonomous Region, China. Eligible patients must comply with the inclusion and exclusion criteria. Participants were recruited through multimodal strategies, including print advertisements, WeChat, volunteer recommendation, and community publicity. All participants will undergo a baseline evaluation, including complete sociodemographic and clinical data collection. Meanwhile, all participants will be invited to voluntarily sign the informed consent.

2.4. Diagnostic Criteria. Compiling Guidelines for Diagnosis and Treatment of Vascular Cognitive Impairment with Reference to the Neurological Society of Chinese Medical Association [18].

(1) Cognitive impairment: the main complaint or informed person-reported cognitive impairment, and objective examination also had evidence of cognitive impairment, and/or objective examination confirmed that cognitive function was lower than before. (2) Vascular factors: including vascular risk factors, history of stroke, focal signs of nervous system, and evidence of cerebrovascular disease shown by imaging, not necessarily all of the above. (3) There is a causal relationship between cognitive impairment and vascular factors. (4) The daily ability is basically normal, but the complex instrumental daily ability can have slight damage. (5) It does not meet the diagnostic criteria of dementia.

2.5. Inclusion Criteria. Patients aged 50-75 years who (1) meet the diagnostic criteria of VCIND described above; (2) MoCA score of 18-26 [19, 20]; (3) are right-handed; (4) elapsed time after stroke $\geq 6$ months; (5) have no acupuncture treatment in the last six months; and (6) volunteer to participate in the research and sign the informed consent were included in the study.

2.6. Exclusion Criteria. Patients who (1) have awareness disorders, severe visual, hearing, and aphasia, and other health assessment failures; (2) have memory and executive dysfunction caused by vascular dementia, Alzheimer's disease, hypothyroidism, brain trauma, etc.; (3) have comorbidity including tumors and severe heart, liver, kidney, hematopoietic system, and endocrine system diseases; (4) have psychiatric history, such as personality disorders and schizophrenia; and (5) have cognitive impairment caused by depression, Baker Depression Scale (>10 points) will be excluded from the study.

2.7. Dropout Criteria. Patients who (1) have serious adverse events, which are not advisable to continue the experiment;
(2) have a secondary stroke after enrollment; and (3) quit the RCT voluntarily will be considered as having dropped out.

2.8. Randomization and Allocation. After meeting the selection criteria, 140 eligible participants will be randomly assigned to the EA group and the CG group in a $1: 1 \mathrm{al}-$ location ratio. The random grouping sequence will be performed according to a random list of numbers generated by the randomization center of Ningxia Medical University and hidden by the opaque and closed envelope. The random sequence will be managed by specific personnel who contact no participant and are not involved in the data collection or analysis.

All participants will receive the following free services, including assessment, functional magnetic resonance imaging (both EA group and CG group), health education (both EA group and CG group), and EA treatment (only EA group) and should adhere to all assessment and treatment schedules as much as possible.

2.9. Blinding. A single-blind method will be used. Outcome assessors, data manager, and statistics analyzer will be blinded to group allocation. However, participants will not be blinded because each participant knows his or her group. Therefore, the results of the assessment will be kept confidential to the participants to improve the accuracy of the test indicators and assure the objectivity and reliability of the evaluation. Blinding will be maintained during the whole study process. After completion of the statistical analysis, the blind code will be disclosed.

\section{Treatment}

3.1. Basic Treatment. Based on their current medical history, all participants received routine treatment with essential drugs, including hypotension, hypoglycemia, antiplatelet aggregation, and lipid regulation.

3.2. Interventions. All participants (2 groups) will be given health education once a week understanding the management of risk factors such as hypertension, diabetes, hyperlipidemia, and obesity and the drug management of these diseases. In addition, participants in the EA group will receive electroacupuncture treatment five sessions a week. Participants will be intervened in screen-separated personal space and will not be allowed to communicate with each other. Treatment will be conducted within 8 consecutive weeks.

3.3. Control Group. The CG group will only receive health education without electroacupuncture treatment. After finishing this trial, electroacupuncture treatment can be freely provided if they need, which is the same as the EA group.

3.4. Electroacupuncture Group. Electroacupuncture treatment details are presented in Table 2 and are based on the revised Standards for Reporting Interventions in Clinical 
TABLE 2: STRICTA checklist (details of intervention).

\begin{tabular}{|c|c|c|}
\hline & Item & Detail \\
\hline \multirow{3}{*}{$\begin{array}{l}\text { 1. Acupuncture } \\
\text { rationale }\end{array}$} & (1a) Style of acupuncture & $\begin{array}{l}\text { Electroacupuncture based on traditional Chinese } \\
\text { medicine theory }\end{array}$ \\
\hline & $\begin{array}{l}\text { (1b) Reasoning for treatment provided, based on } \\
\text { historical context, literature sources, and/or consensus } \\
\text { methods, with references where appropriate }\end{array}$ & $\begin{array}{l}\text { Acupuncture points to be used in this study were Baihui } \\
\text { (GV20) and Shenting (GV24) located at the head. Based } \\
\text { on previous studies [21] and a large number of literature } \\
\text { [22, 23], Baihui (GV20) and Shenting (GV24) will be } \\
\text { mainly selected as acupuncture points for VCI treatment. }\end{array}$ \\
\hline & (1c) Extent to which treatment was varied & Only EA group will receive electroacupuncture treatment \\
\hline \multirow{7}{*}{ 2. Details of needling } & $\begin{array}{l}\text { (2a) Number of needle insertions per subject per } \\
\text { session }\end{array}$ & 2 \\
\hline & (2b) Names of points used & Baihui (GV20) and Shenting (GV24) \\
\hline & $\begin{array}{l}\text { (2c) Depth of insertion, based on a specified unit of } \\
\text { measurement }\end{array}$ & 0.5 inches deep \\
\hline & (2d) Response sought & "De qi" sensation \\
\hline & (2e) Needle stimulation & Electroacupuncture \\
\hline & (2f) Needle retention time & 30 minutes \\
\hline & (2g) Needle type & $\begin{array}{l}\text { Sterilized stainless steel needle with the } 0.3 * 25 \mathrm{~mm} \\
\text { (Suzhou Medical Supplies Factory Co., Ltd., Jiangsu, } \\
\text { China) }\end{array}$ \\
\hline \multirow{2}{*}{ 3. Treatment regimen } & (3a) Num & 40 sessions (8 consecutive weeks) \\
\hline & (3b) Frequency and duration of treatment sessions & 30 minutes per day, 5 days per week \\
\hline \multirow{2}{*}{$\begin{array}{l}\text { 4. Other components } \\
\text { of treatment }\end{array}$} & $\begin{array}{l}\text { (4a) Details of other interventions administered to the } \\
\text { acupuncture group }\end{array}$ & $\begin{array}{l}\text { (a)All participants received routine treatment with } \\
\text { essential drugs(b)All participants will be given health } \\
\text { education once a week }\end{array}$ \\
\hline & $\begin{array}{l}\text { (4b) Setting and context of treatment, including } \\
\text { instructions to practitioners and information and } \\
\text { explanations to patients }\end{array}$ & $\begin{array}{l}\text { The study will be conducted in the General Hospital of } \\
\text { Ningxia Medical University, and all information will be } \\
\text { provided to the subjects }\end{array}$ \\
\hline $\begin{array}{l}\text { 5. Practitioner } \\
\text { background }\end{array}$ & (5) Description of participating acupuncturists & $\begin{array}{l}\text { A medicine acupuncture after completing } 5 \text { years of } \\
\text { Chinese medicine undergraduate course who has more } \\
\text { than } 5 \text { years of clinical experience }\end{array}$ \\
\hline $\begin{array}{l}\text { 6. Control or } \\
\text { comparator } \\
\text { interventions }\end{array}$ & $\begin{array}{l}\text { (6a) Rationale for the control or comparator in the } \\
\text { context of the research question, with sources that } \\
\text { justify this choice } \\
\text { (6b) Precise description of the control or comparator. } \\
\text { If sham acupuncture or any other type of } \\
\text { acupuncture-like control is used, provide details as for } \\
\text { items } 1 \text { to } 3 \text { above }\end{array}$ & $\begin{array}{l}\text { The control group was not treated with sham acupuncture } \\
\text { or placebo acupuncture }\end{array}$ \\
\hline
\end{tabular}

STRICTA: Standards for Reporting Interventions in Clinical Trials of Acupuncture. EA: electroacupuncture.

Trials of Acupuncture (STRICTA) 2010 checklist [24]. In the sitting position, wipe Baihui and Shenting with alcohol, then obliquely pierce the steel needle (Suzhou Medical Supplies Factory Co., Ltd., Jiangsu, China) with the $0.3 * 25 \mathrm{~mm}$ into the skin of the head 0.5 inches deep, and then perform appropriate operations at each point (including lifting, thrusting, and rotating) for 10 seconds to achieve the needling sensation (De qi sensation). Finally, these two needles will be connected to a SDZ-II Huatuo Electroacupuncture Instrument (Hengshui Hengkang Medical Equipment Co., Ltd., Hebei, China) with electrode clamps for 30 minutes per day, 5 days per week, 8 consecutive weeks.

3.5. Outcome Assessment. All outcomes will be measured by several experienced assessors who have been trained to administer these assessments and are blinded to the randomization group after the baseline visit for evaluation. Meanwhile, ensure that each subject's pre- and postevaluations are conducted by the same assessor.
3.6. Primary Outcome. The primary outcome of this study is the changes in the score on MoCA from baseline to 8 weeks after intervention between the two groups and within groups. It is widely used for cognitive function assessment and can reflect the whole cognitive level of individuals. The MoCA has a maximum score of 30 points divided into eight parts, namely, visual space/executive ability ( 5 points), name (3 points), memory (5 points), attention (3 points), computing ( 3 points), language fluency ( 3 points), abstraction ( 2 points), and orientation (6 points). MoCA has excellent reliability and validity and is sensitive enough for clinical and research practice [25].

3.7. Secondary Outcomes. Secondary outcomes include Auditory Verbal Learning Test (AVLT), Test of Attentional Performance (TAP), Stroop color-naming condition (STROOP), Rey-Osterrieth Complex Graphics Testing (ROCFT), and rs-fMRI, all of which will be evaluated at the same time points as the assessment of the primary outcome. 
In this study, the AVLT will be used to evaluate language learning and memory function, which include three tests: immediate recall, short-term delayed recall, and long-term delayed recall [26].

Attention testing is accomplished by the TAP 2.3 attention testing system of PSYTEST Company to test attention span, duration, and functions mainly, which includes four test items: alertness, transfer attention, distracting attention, and response/nonresponse [27].

The STROOP compiled by prime psychological programming software consists of three parts: neutral test, consistency test, and reverse test. It mainly tests the executive function [28].

The visuospatial function was tested by ROCFT, which included the assessment of visual-spatial structure and visual memory [29].

Rs-fMRI will be performed using Philips Ingenia 3.0 T magnetic resonance imager. The data will be scanned by the Department of Radiology, General Hospital of Ningxia Medical University. The specific operation scheme is as follows.

3.8. Brain MRI Protocol. For participants, the imaging data which include conventional brain T1-weighted image (T1WI) and resting fMRI will be collected and acquired using a 3.0 T Ingenia scanner (Philips, the Netherlands) at the General Hospital of Ningxia Medical University. T1weighted sequence and resting fMRI are shown as the following parameters, respectively [21].

T1: repetition time $(\mathrm{TR})=8.1 \mathrm{~ms}$, echo time $(\mathrm{TE})=3.7 \mathrm{~ms}$, flip angle $(\mathrm{FA})=8^{\circ}$, number of slices $=150$, slice thickness $=1 \mathrm{~mm}$, voxel size $=1 \times 1 \times 1 \mathrm{~mm}^{3}, \quad F O V=240 \mathrm{~mm} \times$ $240 \mathrm{~mm}$, and matrix $=240 \times 222$.

Resting fMRI: $\mathrm{TR}=2000 \mathrm{~ms}, \mathrm{TE}=30 \mathrm{~ms}$, flip angle $=90^{\circ}$, number of slices $=36$, slice thickness $=3.8 \mathrm{~mm}$, voxel size $=3.01 \times 3.09 \times 3.80 \mathrm{~mm}^{3}, \quad F O V=241 \mathrm{~mm} \times 241 \mathrm{~mm}$, matrix $=80 \times 78$, and phases per location $=240$.

Participants will be requested to lie quietly in the scanner and close their eyes without thinking during the data acquisition process. Meanwhile, earplugs will be utilized to reduce the noise when the machine is running. Each scan lasted 1380 seconds.

3.9. Data Collection, Management, and Monitoring. Well-designed electronic CRF tables will be used for data collection. During the data collection process, a dependent research assistant (RA) will responsible for quality control. Two independent researchers will check the Case Report Form and Adverse Events Form carefully and input them into the computer to ensure data accuracy.

The project manager will have access to the data and be responsible for the initial data cleansing, identification, coding, and conversion to the appropriate data analysis format. All the original forms will be archived in the clinical research center of the General Hospital of Ningxia Medical University. Then, all data are treated with the utmost confidentiality and made anonymous to anyone outside the study.
The Data and Safety Monitoring Board (DSMB) of the clinical evaluation center of the General Hospital of Ningxia Medical University are responsible for data monitoring, i.e., obtaining any interim results and making the final decision to terminate the trial if any serious acupuncture-related EA occurs.

3.10. Statistical Analysis. IBM SPSS Statistics 24.0 software will be used to conduct the whole data analysis by an independent statistician who is unaware of group assignments. All continuous variables will be presented as mean \pm standard deviation (SD), and categorical variables will be presented as numbers (\%). The statistical significance level is considered to be $95 \%$ CIs (two-sided alpha $P<0.05$ ).

The effect of the intervention will be assessed as the change in outcome measures between the baseline and eight weeks (after treatment sessions end), using a repeatedmeasures analysis of variance (ANOVA) and chi-square test.

The difference in effect between the CG group and the EA group will be assessed by comparing changes in the MoCA score and secondary outcomes including language learning and memory function, attention function, executive function, and visuospatial function using the independent $t$ test or Mann-Whitney $U$ test on continuous variables and chi-square test or Fisher's exact test on categorical variables.

Intention-to-treat analyses will be used for all outcomes. A multiple imputation method will be carried out to fill in missing data for outcomes.

The structure and function of functional magnetic resonance will be analyzed by SPM software based on the MATLAB platform. Neural network analysis techniques such as VBM, ROI, and ICA will be used to compare the differences in functional connectivity changes between groups by independent sample $t$-test. FDR correction will be used for multiple comparison and correction.

3.11. Quality Control. To ensure the quality of this trial, the DSMB of the clinical evaluation center of the General Hospital of Ningxia Medical University will monitor regularly and strictly, consisting of informed consent, participant screening, intervention, serious EA records, statistical analysis, and data management.

3.12. Investigation of Adverse Events and Safety. During the acupuncture treatment, subjects will be informed about adverse events such as local bleeding, subcutaneous hematoma, or pain associated and serious adverse events such as dizziness and palpitation in advance. Any adverse events and serious adverse events during treatment will be reported in the Case Report Form (CRF) and investigated, including its type and frequency, and appropriate treatments for the associated symptoms will be provided immediately to minimize serious adverse events. If a serious adverse event occurs, the case will be reported to the Data and Safety Monitoring Board (DSMB) of the clinical evaluation center of the General Hospital of Ningxia Medical University immediately and the blinding code will be broken. If 
necessary, even part or all of the trial will be suspended until further instructions are expected to be available. We will pay for the treatment of the subjects with serious adverse events until recovery.

3.13. Trial Status. The project is ongoing recruitment and intervention.

\section{Discussion}

Governor vessel is one of the important meridians of the human body, which has the function of Governor Yang Qi and regulating spirit. Baihui and Shenting are very important acupoints on the Governor vessel. Baihui is the place where Yang Qi of the Governor vessel at its peak and Shenting is the place where wisdom is concentrated. Combining the stimulation of the two acupoints can strengthen the function of regulating the Du meridian and the spirit. Considering the importance of Du Meridian, Baihui and Shenting acupoints will be mainly selected in the treatment of cognitive disorders in traditional Chinese medicine, based on previous studies [22] and a large number of literature [23, 30].

On the other hand, fewer serious adverse events indicate that acupuncture is one of the safest nonpharmaceutical treatments [31, 32]. Although the clinical effectiveness of EA in improving cognitive impairment and/or major symptoms of dementia has been established, the central mechanism of EA is not clear yet. Neuroimaging, as an effective means to explore the central mechanism of acupuncture's effects, is widely used in acupuncture research [33]. Resting-state functional connectivity of the brain network reflects the inherent and spontaneous neural activity of the brain activity pattern; it is one of the most important technologies for assessing brain function and has good clinical applicability.

Based on the previous literature, acupuncture could increase DMN connectivity with pain-, affective-, and memory-related brain areas $[34,35]$, but evidence in vascular cognitive impairment is lacking. For this reason, we chose EA to intervene in VCIND, to explore its clinical efficacy and central mechanism, especially the changes of brain DMN.

Some limitations of this study should be noted. First, this trial is only one RCT without follow-up; whether these patients will convert to VD in the future is uncertain. Therefore, a follow-up to track the dynamic evolution of these patients is planned. Second, there is no therapeutic comparison in our study. We only set up a blank comparison group for observation. Therefore, we plan to establish a sham acupuncture group or drug group in future studies. Third, this trial is also restricted to a single center.

In conclusion, the results of this study following the CONSORT [36] and STRICTA [24] guidelines are expected to elucidate the clinical effects and neuroimaging mechanism of EA for VCIND, providing more solid evidence for traditional Chinese medicine (TCM) practitioners to give EA treatment for VCIND. Besides, we hope that our further research study will be improved and carried out in multicentered hospitals with an expanded sample size according to these experiences.

\section{Abbreviations}

VCIND: Vascular cognitive impairment no dementia

EA: $\quad$ Electroacupuncture

VD: Vascular dementia

CG: $\quad$ Control group

MoCA: Montreal Cognitive Assessment

AVLT: Auditory Verbal Learning Test

STROOP: Stroop color-naming condition

ROCFT: Rey-Osterrieth Complex Graphics Testing

rs-fMRI: Resting-state functional magnetic resonance imaging.

\section{Data Availability}

Data and materials are available upon request from the corresponding author.

\section{Additional Points}

Recruitment began in August 2018 (Protocol version 1.0).

\section{Ethical Approval}

This study followed the Declaration of Helsinki exactly. This trial was subject to approval by the Ethics Board of the General Hospital of Ningxia Medical University (2018-239).

\section{Consent}

All participants were completely informed of the study and signed the informed consent before participation.

\section{Disclosure}

The trial was registered at the Chinese Clinical Trial Registry on 28 July 2018 (ChiCTR1800017398). The funding body had no role in the study design or the decision to submit the manuscript for publication.

\section{Conflicts of Interest}

The authors declare that they have no conflicts of interest.

\section{Authors' Contributions}

$\mathrm{RL}$ and JH contributed equally to this work and designed the trial. RL drafted the manuscript. Ruizhu Lin and Jia Huang designed the trial. Ruizhu Lin wrote the first draft. Jianfeng $\mathrm{Xu}$, Jing Tao, Ying Xu, Jiao Liu, Weilin Liu, Shengxiang Liang, Minguang Yang, and Lidian Chen were responsible for critical revision of the draft. All authors read and approved the final manuscript. 


\section{Acknowledgments}

This study was supported by the Rehabilitation Research Center of Fujian Province and Rehabilitation Research Center for Traditional Chinese Medicine, State Administration of Traditional Chinese Medicine, for the People's Republic of China. This research was funded by the Rehabilitation Innovation Cooperative Center of Fujian University of Traditional Chinese Medicine, China (grant no. X2018001).

\section{Supplementary Materials}

SPIRIT 2013 checklist: recommended items to address in a clinical trial protocol and related documents. (Supplementary Materials)

\section{References}

[1] V. Hachinski, C. Iadecola, R. C. Petersen et al., "National institute of neurological disorders and stroke-Canadian stroke network vascular cognitive impairment harmonization standards," Stroke, vol. 37, no. 9, pp. 2220-2241, 2006.

[2] A. Ritter and J. A. Pillai, "Treatment of vascular cognitive impairment," Current Treatment Options in Neurology, vol. 17, no. 8, p. 367, 2015.

[3] A. M. Helman and M. P. Murphy, "Vascular cognitive impairment: modeling a critical neurologic disease in vitro and in vivo," Biochimica et Biophysica Acta (BBA)-Molecular Basis of Disease, vol. 1862, no. 5, pp. 975-982, 2016.

[4] M. Wiesmann, A. J. Kiliaan, and J. A. Claassen, "Vascular aspects of cognitive impairment and dementia," Journal of Cerebral Blood Flow \& Metabolism, vol. 33, no. 11, pp. 1696-1706, 2013.

[5] B. Jiang, C. Ding, G. Yao et al., "Polysomnographic abnormalities in patients with vascular cognitive impairment-no dementia," Sleep Medicine, vol. 14, no. 11, pp. 1071-1075, 2013.

[6] Y. Zeng, Y. Bao, M. Zhu, S. Chen, and J. Fang, "Mild cognitive impairment of stroke at subacute stage treated with acupuncture: a randomized controlled trial," Zhongguo Zhen Jiu, vol. 35, no. 10, pp. 979-982, 2015.

[7] J. H. Lu, J. Tan, H. Zhang, and Y. Q. Yuan, "Meta-analysis of acupuncture clinical efficacies on ischemic type cognitive dysfunction," Guiding Journal of Traditional Chinese Medicine and Pharmacology, vol. 21, no. 20, pp. 87-89, 2015.

[8] H. Zhang, L. Zhao, S. Yang et al., "Clinical observation on effect of scalp electroacupuncture for mild cognitive impairment," Journal of Traditional Chinese Medicine, vol. 33, no. 1, pp. 46-50, 2013.

[9] E. J. Yang, M. Cai, and J.-H. Lee, "Neuroprotective effects of electroacupuncture on an animal model of bilateral common carotid artery occlusion," Molecular Neurobiology, vol. 53, no. 10, pp. 7228-7236, 2016.

[10] H. H. Shen, "Core concept: resting-state connectivity," Proceedings of the National Academy of Sciences, vol. 112, no. 46, pp. 14115-14116, 2015.

[11] J. Wang, Y. Chen, H. Liang et al., "The role of disturbed smallworld networks in patients with white matter lesions and cognitive impairment revealed by resting state function magnetic resonance images (rs-fMRI)," Medical Science Monitor, vol. 25, pp. 341-356, 2019.

[12] R. L. Buckner, J. R. Andrews-Hanna, and D. L. Schacter, "The brain's default network: anatomy, function, and relevance to disease," Annals of the New York Academy of Sciences, vol. 1124, no. 1, pp. 1-38, 2008.

[13] Y. Chen, C. Wang, H. Liang et al., "Resting-state functional magnetic resonance imaging in patients with leukoaraiosisassociated subcortical vascular cognitive impairment: a crosssectional study," Neurological Research, vol. 38, no. 6, pp. 510-517, 2016.

[14] R.-L. Cai, G.-M. Shen, H. Wang, and Y.-Y. Guan, "Brain functional connectivity network studies of acupuncture: a systematic review on resting-state fMRI," Journal of Integrative Medicine, vol. 16, no. 1, pp. 26-33, 2018.

[15] Y. Shi, S. Zhang, Q. Li et al., "A study of the brain functional network of Deqi via acupuncturing stimulation at BL40 by rsfMRI," Complementary Therapies in Medicine, vol. 25, pp. 71-77, 2016.

[16] A.-W. Chan, J. M. Tetzlaff, P. C. Gøtzsche et al., "SPIRIT 2013 explanation and elaboration: guidance for protocols of clinical trials," BMJ, vol. 346, Article ID e7586, 2013.

[17] M. Liao, N. Dong, and Z. Chen, "Effect of electroacupuncture on patients with non dementia vascular cognitive impairment," Journal of Clinical Acupuncture and Moxibustion, vol. 32, no. 3, pp. 46-48, 2016.

[18] The Writing Group of the Dementia and Cognitive Society of Neurology Committee of Chinese Medical Association, "Chinese medical association: guidelines for the diagnosis and treatment of vascular cognitive impairment," Chinese Journal of Neurology, vol. 44, no. 2, pp. 142-147, 2011.

[19] Z. S. Nasreddine, N. A. Phillips, V. R. Bédirian et al., "The montreal cognitive assessment, MoCA: a brief screening tool for mild cognitive impairment," Journal of the American Geriatrics Society, vol. 53, no. 4, pp. 695-699, 2005.

[20] J. Peng, Z. L. Liu, and Z. WJ, "Study of cognitive function scale in patients with vascular cognitive impairment no dementia," Chinese Journal of Brain Diseases and Rehabilitatin (Electronic Edition), vol. 4, no. 5, pp. 305-310, 2014.

[21] C. L. Hsu, J. R. Best, M. W. Voss et al., "Functional neural correlates of slower gait among older adults with mild cognitive impairment," The Journals of Gerontology: Series A, vol. 74, no. 4, pp. 513-518, 2019.

[22] J. Wu, J. Wang, and J. Zhang, "Theoretic basis on the same therapeutic program for different degenerative brain diseases in terms of the Governor vessel: Alzheimer's disease and Parkinson's disease," Zhongguo Zhen Jiu, vol. 35, no. 5, pp. 489-492, 2015.

[23] F. Liu, L. Q. Yao, and J. H. Chen, "Therapeutic efficacy of acupuncture at Baihui (GV 20) and shenting (GV 24) for poststroke cognitive impairment: 20-a systematic review," Shanghai Journal of Acupuncture and Moxibustion, vol. 37, no. 1, pp. 104-111, 2018.

[24] H. MacPherson, D. G. Altman, R. Hammerschlag et al., "Revised standards for reporting interventions in clinical trials of acupuncture (STRICTA): extending the CONSORT statement," Acupuncture and Related Therapies, vol. 3, no. 4, pp. 35-46, 2015.

[25] S. Hoops, S. Nazem, A. D. Siderowf et al., "Validity of the MoCA and MMSE in the detection of MCI and dementia in Parkinson disease," Neurology, vol. 73, no. 21, pp. 1738-1745, 2009.

[26] D. W. Loring, S. C. Bowden, E. Staikova, J. A. Bishop, D. L. Drane, and F. C. Goldstein, "NIH toolbox picture sequence memory test for assessing clinical memory function: diagnostic relationship to the Rey auditory verbal learning test," Archives of Clinical Neuropsychology, vol. 34, no. 2, pp. 268-276, 2019. 
[27] J. A. Mortimer, D. Ding, A. R. Borenstein et al., "Changes in brain volume and cognition in a randomized trial of exercise and social interaction in a community-based sample of nondemented Chinese elders," Journal of Alzheimer's Disease, vol. 30, no. 4, pp. 757-766, 2012.

[28] F. Scarpina and S. Tagini, "The Stroop color and word test," Frontiers in Psychology, vol. 8, p. 557, 2017.

[29] B. F. Dias, L. O. Rezende, L. F. Malloy-Diniz, and J. J. d. Paula, "Relationship between visuospatial episodic memory, processing speed and executive function: are they stable over a lifespan?" Arquivos de Neuro-Psiquiatria, vol. 76, no. 2, pp. 89-92, 2018.

[30] F. Yang, K.-T. Luo, J.-H. Zhu, and Y.-M. Xu, "Effect of longtime needle retaining at Baihui (GV 20) on cognitive impairment in post-stroke patients," Journal of Acupuncture and Tuina Science, vol. 15, no. 6, pp. 398-402, 2017.

[31] H. Zhao, D. Li, Y. Li et al., "Efficacy and safety of acupuncture for hypertension: an overview of systematic reviews," Complementary Therapies in Clinical Practice, vol. 34, pp. 185-194, 2019.

[32] J. Y. Tan, A. Molassiotis, T. Wang, and L. K. Suen, “Adverse events of auricular therapy: a systematic review," EvidenceBased Complementary and Alternative Medicine, vol. 2014, Article ID 506758, 20 pages, 2014.

[33] C. Rong-lin, S. Guo-ming, W. Hao, and G. Yuan-yuan, "Brain functional connectivity network studies of acupuncture: a systematic review on resting-state fMRI," Journal of Integrative Medicine, vol. 16, no. 1, pp. 26-33, 2018.

[34] X. Niu, M. Zhang, Z. Liu et al., "Interaction of acupuncture treatment and manipulation laterality modulated by the default mode network," Molecular Pain, vol. 13, Article ID 2072479836, 2017.

[35] Y. Zhang, H. Zhang, T. Nierhaus, D. Pach, C. M. Witt, and M. Yi, "Default mode network as a neural substrate of acupuncture: evidence, challenges and strategy," Frontiers in Neuroscience, vol. 13, p. 100, 2019.

[36] K. F. Schulz, D. G. Altman, and D. Moher, "CONSORT 2010 statement: updated guidelines for reporting parallel group randomised trials," International Journal of Surgery, vol. 9, no. 8, pp. 672-677, 2011. 